\title{
Identifying the variables associated with pain during transrectal ultrasonography of the prostate
}

This article was published in the following Dove Press journal:

Patient Preference and Adherence

24 August 2015

Number of times this article has been viewed

\section{Chen-Pang Hou ${ }^{1,2}$ \\ Yu-Hsiang Lin ${ }^{1,2}$ \\ Meng-Chiao $\mathrm{Hsieh}^{3}$ \\ Chien-Lun Chen ${ }^{1,2}$ \\ Phei-Lang Chang ${ }^{1,2}$ \\ Ying-Chen Huang ${ }^{2}$ \\ Ke-Hung Tsui ${ }^{1,2}$}

'Department of Urology, Chang Gung Memorial Hospital at Linkou, ${ }^{2} \mathrm{~S} c h o o l$ of Medicine, Chang Gung University, ${ }^{3}$ Division of Colon and Rectal Surgery, Department of Surgery, Chang Gung Memorial Hospital at Chiayi, Chang Gung University, Kwei-Shan, Tao-Yuan, Taiwan
Correspondence: Ke-Hung Tsui

Department of Urology, Chang Gung Memorial Hospital at Linkou, Chang Gung University, 5 Fu-Shing Street, Kwei-Shan, Tao-Yuan 333, Taiwan

Tel +886 3328 I 200 Extension 2137

Fax +886227358775

Email khtsui@yahoo.com
Objective: The purpose of this study was to prospectively investigate the degree of pain experienced by the patients receiving transrectal ultrasonography (TRUS) of the prostate by applying a visual analog scale. We also identified the clinical parameters influencing pain during the TRUS examination.

Materials and methods: Records were obtained from a prospective database for male patients who received TRUS of prostate in the outpatient department of Chang Gung Memorial Hospital, Taiwan, from January 2014 to June 2014. The patients underwent a detailed physical examination and medical history review. Immediately after the TRUS examination, the patients completed questionnaires based on a ten-point visual analog pain scale. The variables of interest were age, body mass index, prostate volume, prostate sagittal length, prostate-specific antigen, previous TRUS experience, external hemorrhoids, anal surgical history, prostate calcification, and image artifact caused by stool in the rectum. All variables were correlated to the visual analog scale by applying multivariate regression analysis.

Results: By using linear regression analysis, we identified the independent factors that affected the pain score during the TRUS examination. The patients who received the examination for the first time or had longer prostate sagittal lengths, external hemorrhoids, anal surgical history, or stool stored in the rectum experienced more pain during the TRUS examination. Furthermore, the pain was reduced when we provided the patients with a detailed explanation before the procedure and allowed them to observe the real-time images during the examination.

Conclusion: Although a TRUS examination is uncomfortable for patients, after having identified the factors affecting pain, physicians can assist patients in reducing pain during the procedure, thus providing higher quality examinations.

Keywords: VAS, TRUS, ultrasound, pain score, transrectal, benign prostate hyperplasia, digital examination

\section{Introduction}

The first clinically applicable images of the prostate obtained through transrectal ultrasonography (TRUS) were described in 1967 by Watanabe et al. ${ }^{1}$ Because ultrasound technology has become more refined over the years, the use of TRUS to evaluate prostatic disease has increased. By the mid-1980s, the $7 \mathrm{MHz}$ ultrasound probe, which clearly delineates the architecture of the prostate, had become a standard diagnostic instrument. ${ }^{2}$ TRUS can be used to detect prostate abnormalities such as benign prostatic hyperplasia, carcinoma of the prostate, prostatitis, prostatic abscess, and prostatic calculi. ${ }^{3}$ However, a TRUS examination of the prostate is uncomfortable, ${ }^{4}$ and some patients at Chang Gung Memorial Hospital have claimed that they experienced severe pain when receiving this invasive procedure. Although the degree of and factors influencing pain during TRUS-guided biopsy have been discussed in the literature, ${ }^{5-7}$ based on our research, no study has focused on the pain experienced by patients 
receiving TRUS of the prostate. The purpose of this study was to determine the manner in which patients experience the pain, and to identify the clinical parameters influencing the pain during a TRUS examination.

\section{Materials and methods}

This study was approved by the Institutional Review Board at Chang Gung Memorial Hospital, Taiwan. Records were obtained from a prospective database for male patients who received TRUS of the prostate in the outpatient department from January 2014 to June 2014. The patients underwent a detailed medical history review and physical examination. The patient's age, body mass index, prostate-specific antigen level, previous anal operative history, and TRUS experience were recorded. When routine digital rectal examinations were performed, the anus was carefully inspected and the presence of external hemorrhoids was recorded. Patients with dementia, disabilities that interfered with verbal communication or neurological diseases that potentially influenced pain assessment, as well as patients who had prostatitis or who had received transurethral resection of the prostate were excluded. A total of 498 patients were enrolled in the study.

The patients were randomized into two groups according to medical chart number (odd, group 1; even, group 2). The group 1 patients received the TRUS examination according to the routine procedure of the hospital. By contrast, the group 2 patients received a detailed explanation of the examination procedure immediately before the examination began. In addition, a monitor was placed next to them, enabling them to observe the real-time images during the entire examination. Transrectal ultrasound was performed on the patients by a single urologist using an ultrasound machine (Prosound Alpha 6, Hitachi Ltd., Tokyo, Japan) fitted with a 7.5 MHz biplanar transrectal probe. The prostate volume was measured using the ellipse formula (sagittal length $\times$ width $\times$ height $\times 0.52$ ). Whether the patients had stool stored in the rectum that interfered with the image quality (Figure 1) was recorded. Immediately following the examination, the patients completed a visual analog scale (VAS) questionnaire, evaluating the pain experienced during the TRUS on a scale of $0-10$. After the patients rated the pain by marking a line to indicate pain level, a rounding method was used to obtain an integer.

The pain score distribution was examined using the Kolmogorov-Smirnov test. The $\chi^{2}$ test was applied to test qualitative data, and the Mann-Whitney $U$-test was used to test numerical variables. All aforementioned variables were analyzed through linear regression analysis to identify the independent factors influencing the pain experienced during the procedure.

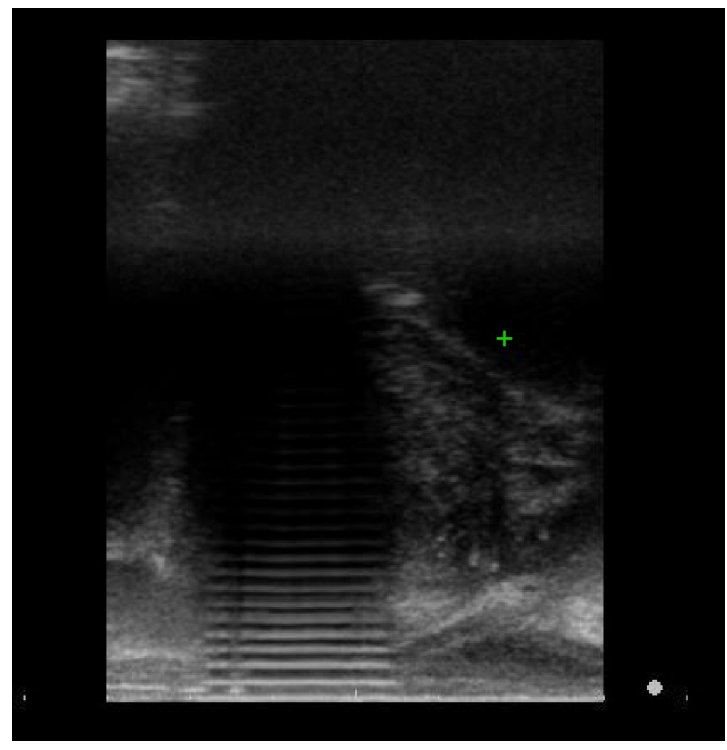

Figure I Image shows artifact caused by stool stored in the rectum and interfered with the image quality. The examiner cannot easily measure the prostate sagittal length.

\section{Results}

The clinical characteristics of the patients are summarized in Table 1. The median patient age, prostate volume, and mean pain score was 63 years (range: $43-87$ ), $32.40 \mathrm{~g}$ (range: 10.3-162.00), and 2.87 (range: 0-9), respectively. The distribution of the pain score is presented in Figure 2. No pain (VAS $=0$ ) was reported by 53 patients $(10.64 \%$ ), mild pain (VAS $=1-3$ ) was described by 318 patients $(63.86 \%)$, and moderate pain (VAS $=4-5$ ) was indicated by $107(21.49 \%)$ patients. However, 60 patients (12.05\%) reported experiencing severe pain (VAS $=6-10$ ) during the examination. The normal distribution of this result was confirmed through the Kolmogorov-Smirnov test (Figure 3).

When the patients were further subgrouped into two groups: VAS $\leq 3$ and VAS $\geq 4$, as shown in Table 2, we found that the patients who indicated VAS scores $\geq 4$ were younger (63.04 vs $65.79, P=0.0293$ ), had a larger prostate volume (43.04 g vs $37.41 \mathrm{~g}, P=0.0161$ ), and longer prostate sagittal length ( $4.69 \mathrm{~cm}$ vs $4.56 \mathrm{~cm}, P=0.0415)$. The data

Table I Characteristics of the study population

\begin{tabular}{ll}
\hline Variable (number =498) & n (range) \\
\hline Median age (years) & $63(43-87)$ \\
Median BMI & $24.98(16.8-47.06)$ \\
Median PSA $(\mathrm{ng} / \mathrm{mL})$ & $5.03(0.1-398.7)$ \\
Median prostate volume $(\mathrm{mL})$ & $32.40(10.3-162.00)$ \\
Median prostatic sagittal length $(\mathrm{cm})$ & $4.50(2.42-7.50)$ \\
Mean pain score $(\mathrm{VAS})$ & $2.87 \pm 2.02(0-9)$ \\
\hline
\end{tabular}

Abbreviations: BMI, body mass index; PSA, prostate-specific antigen; VAS, visual analog scale. 


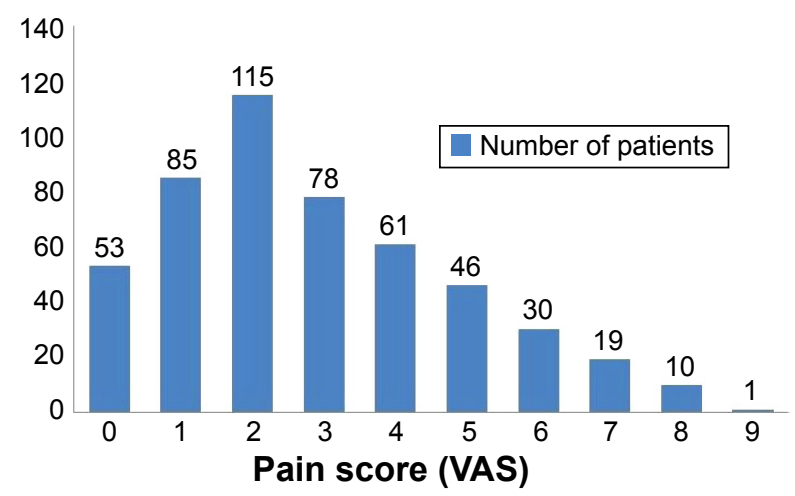

Figure 2 Distribution of pain score. Abbreviation: VAS, visual analog scale.

also revealed that a lower percentage of patients whose VAS scores were $\geq 4$ had TRUS experience ( $13.33 \%$ vs $23.85 \%$, $P=0.0427)$ and were randomly assigned to group $2(34.91 \%$ vs $60.48 \%, P<0.0001)$, compared with patients with VAS scores of $\leq 3$. By contrast, a higher percentage of patients with VAS scores of $\geq 4$ had external hemorrhoids $(59.09 \%$ vs $26.67 \%, P<0.0001)$, anal surgical experience $(31.63 \%$ vs $6.96 \%, P<0.0001)$, and image artifact caused by stool (22.85\% vs $11.79 \%, P=0.0068)$, as shown in Table 3 .

The group 2 patients had lower VAS scores than group 1 patients ( 2.62 vs 3.09, $P=0.002$ ), as shown in Figure 4. In other words, the patients who received a detailed explanation of the procedure and were allowed to observe real-time images experienced less pain compared with the patients who did not.

Clinical parameters associated with TRUS that influenced VAS scores were analyzed (Table 4). Multivariate analysis revealed that the prostate sagittal length was the continuous

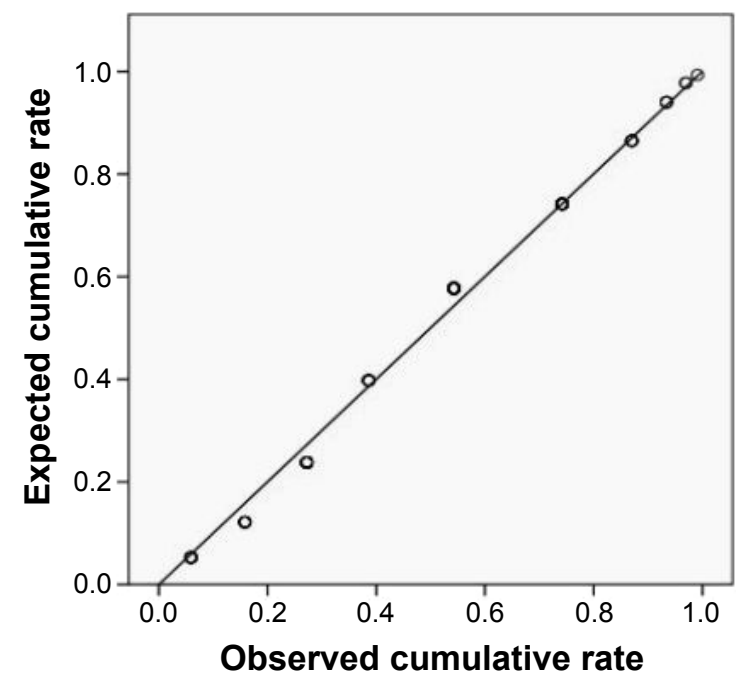

Figure 3 Kolmogorov-Smirnov test results showing that the pain score was normally distributed.
Table 2 Comparison of patients' numerical variables between pain score $\leq 3$ and pain score $\geq 4$

\begin{tabular}{llll}
\hline Characteristics & $\begin{array}{l}\text { Pain score } \leq \mathbf{3} \\
\text { mean } \pm \text { SD }\end{array}$ & $\begin{array}{l}\text { Pain score } \geq \mathbf{4} \\
\text { mean } \pm \text { SD }\end{array}$ & P-value \\
\hline Age (years) & $65.79 \pm 10.92$ & $63.04 \pm 11.67$ & $0.0293^{*}$ \\
BMI & $25.11 \pm 3.86$ & $25.20 \pm 3.76$ & 0.9314 \\
Prostate volume $(\mathrm{gm})$ & $37.4 I \pm 20.39$ & $43.04 \pm 24.45$ & $0.016 I^{*}$ \\
Prostate sagittal & $4.56 \pm 0.78$ & $4.69 \pm 0.94$ & $0.0415^{*}$ \\
length $(\mathrm{cm})$ & & & \\
PSA $(\mathrm{ng} / \mathrm{mL})$ & $3.24 \pm 2.17$ & $4.85 \pm 3.82$ & 0.8216 \\
\hline
\end{tabular}

Notes: Comparisons were made using Mann-Whitney U-test. *Indicates significant difference.

Abbreviations: BMI, body mass index; PSA, prostate-specific antigen; SD, standard deviation.

variable influencing the level of pain. Assignment to group 2 and TRUS experience were negatively associated with the VAS score, whereas external hemorrhoids, anal surgical history, and image artifact caused by stool were independent factors that increased the VAS score.

\section{Discussion}

The VAS is a simple assessment tool consisting of a $10 \mathrm{~cm}$ line with 0 on one end and 10 on the other. ${ }^{8}$ Patients rate their pain by placing a mark on the line that corresponds to their current level of pain. The scale can be used as a baseline assessment of pain and to evaluate treatment effectiveness. ${ }^{8}$ This scale has been widely used in many fields of pain research because it is easy to understand, requires less than 1 minute to complete, and has high validity and reliability. ${ }^{9}$ Therefore, we applied the VAS in our study. Evaluating the degree of pain by using VAS and exploring the factors influencing pain during TRUS-guided biopsy have been discussed in the literature. ${ }^{5-7}$ However, based on our research, no study has focused on the pain experienced by the patients receiving TRUS of the prostate. Although the examination is considered an easy procedure by urologists and requires no local anesthesia, some patients claim to have experienced severe pain during the examination. Therefore, we focused on determining the pain scale distribution of the patients and identifying the factors influencing the pain. Our data revealed that the pain scores experienced by the patients were in a normal distribution, and the mean VAS score was only 2.87 . This indicated that most of the patients tolerated the pain well and required no anesthesia during the procedure.

Our study revealed that patients with VAS scores of $\geq 4$ were younger than the patients with VAS scores of $\leq 3$ (63.04 vs $65.79, P=0.0293$ ). Although multivariate analysis showed that the age was not a strong factor influencing the pain $(P=0.0671$; Table 4$)$, we maintain that age 
Table 3 Comparison of patients' qualitative variables between pain score $\leq 3$ and pain score $\geq 4$

\begin{tabular}{llll}
\hline Characteristics & Pain score $\leq \mathbf{3}(\%)$ & Pain score $\leq \mathbf{4}$ (\%) & P-value \\
\hline Previous TRUS experience (\%) & $23.85 \%$ & $13.33 \%$ & $0.0427^{*}$ \\
Group 2 (\%) & $60.48 \%$ & $34.91 \%$ & $<0.000 I^{*}$ \\
External hemorrhoids (\%) & $26.67 \%$ & $59.09 \%$ & $<0.000 I^{*}$ \\
Prostate calcification (\%) & $22.11 \%$ & $36.19 \%$ & 0.0893 \\
Previous anal operation (\%) & $6.96 \%$ & $31.63 \%$ & $<0.000 I^{*}$ \\
Stool artifact (\%) & $11.79 \%$ & $22.85 \%$ & $0.0068^{*}$ \\
\hline
\end{tabular}

Notes: Comparisons were made using $\chi^{2}$ test. *Indicates significant difference. Abbreviation: TRUS, transrectal ultrasound of the prostate.

plays an influential role. Philips et $\mathrm{al}^{10}$ and Rodriguez et al ${ }^{11}$ have reported that older patients can tolerate pain more during a TRUS-guided prostate biopsy because of a relatively low anal resting tone. The internal anal sphincter provides approximately $60 \%-70 \%$ of the anal resting tone, whereas the external anal sphincter is responsible for $20 \%-30 \%$; the remaining $10 \%-15 \%$ is attributed to expansion of the anal cushions, call hemorrhoids. ${ }^{12-15}$ Young patients have a relatively high resting anal tone and low anorectal compliance during probe insertion and withdrawal. ${ }^{10-11}$ Consequently, younger patients experience more pain during the procedure. Prostate configuration also played a crucial role in the pain during TRUS. Our results revealed that the prostate sagittal length, rather than the prostate volume, affected the pain level. Patients who had a longer prostate sagittal length experienced more pain because when measuring the prostate volume, the probe of the ultrasonography had to be inserted deeper in the rectum, causing more discomfort.

Patients with external hemorrhoids accounted for approximately $33.33 \%$ of the entire study population. Hemorrhoids are categorized as external hemorrhoids (distal to the dentate line), internal hemorrhoids (proximal to the dentate line), and mixed hemorrhoids (both proximal and distal). External hemorrhoids are more sensitive to pain, itching, and irritation than are internal hemorrhoids located proximal to the dental

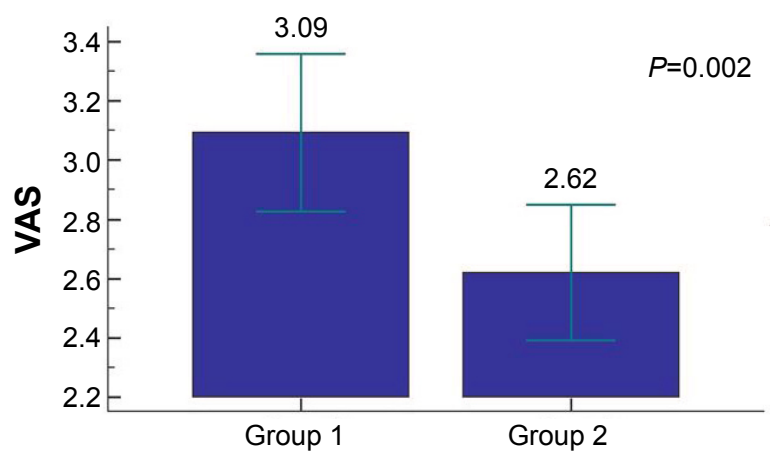

Figure 4 Significantly lower pain scores of group 2 patients compared with those of group I patients.

Abbreviation: VAS, visual analog scale. line. ${ }^{16}$ During the TRUS examination, the external hemorrhoids of patients are irritated and are painful. According to our analysis, a higher VAS score was reported by patients with external hemorrhoids, which was an independent factor influencing the pain (Table 4). In addition, patients who had anal surgical history also reported a higher degree of pain during TRUS examination. Delayed anal scar tissue, fibrosis, asymptomatic stenosis, or even symptomatic stricture may occur after anal surgical procedures. The incidence of late anal stenosis is not too low and occurs in $4 \%-5 \%$ of patients receiving hemorrhoidectomy. ${ }^{17} \mathrm{~A}$ previous study reported that fistulectomy can also induce anal fibrosis. ${ }^{18}$ Although none of our patients mentioned having the aforementioned surgical complications, we determined that an anal surgical history reduced the compliance of the anal sphincter and, consequently, caused more pain during the probe insertion and withdrawal. Therefore, we suggest using a digital finger insertion with ample lubricant to rinse the dry anal canal, gradually dilating the anal sphincter to prevent anal sphincter spasms. The probe can be inserted slowly and gently after the resting anal tone decreases. Using this technique enables reducing the pain experienced during a TRUS examination by patients with external hemorrhoids or anal surgical history. Our study also revealed that patients experienced more pain when they had stool stored in the rectum that caused image artifact, possibly because the examiner had to obtain a higher quality image by shifting and adjusting the ultrasound probe position in the rectum. Therefore, we suggest that patients who have chronic constipation receive an enema before the procedure.

Czarnecki et al mentioned that pain is based on patient perceptions, which may be influenced by interrelated factors including the patient's emotional and psychological state, level of anxiety, previous pain experience, understanding of the procedure, and medical condition and environmental factors. ${ }^{19}$ This may explain why the patients who had previous TRUS experience experienced less pain during the procedure. Furthermore, explaining the examination and allowing 
Table 4 Multivariate analysis for the pain associated with TRUS using linear regression analysis

\begin{tabular}{|c|c|c|c|}
\hline Variable & $\beta$ & SE $(\beta)$ & $P$-value \\
\hline Age (continuous variable) & -0.01381 & 0.0075 & 0.0671 \\
\hline BMI (continuous variable) & -1.001942 & 0.0209 & 0.9258 \\
\hline Prostate volume (continuous variable) & -0.00131 & 0.0020 & 0.6571 \\
\hline $\begin{array}{l}\text { Prostate sagittal length (continuous } \\
\text { variable) }\end{array}$ & 0.005547 & 0.0021 & $0.0072 *$ \\
\hline PSA (continuous variable) & $0.0000283 I$ & $<0.001$ & 0.717 \\
\hline Group 2 (vs group I) & -0.4484 & 0.1570 & $0.0045^{*}$ \\
\hline $\begin{array}{l}\text { Previous TRUS experience (vs absence } \\
\text { of previous TRUS experience) }\end{array}$ & -0.4234 & 0.1932 & $0.0289 *$ \\
\hline $\begin{array}{l}\text { External hemorrhoids (vs absence of } \\
\text { external hemorrhoids) }\end{array}$ & $1.26 \mathrm{I}$ & 0.1736 & $<0.000 I^{*}$ \\
\hline $\begin{array}{l}\text { Previous anal operation history } \\
\text { (vs absence of anal operation history) }\end{array}$ & 1.568 & 0.2476 & $<0.000 I^{*}$ \\
\hline $\begin{array}{l}\text { Prostate calcification (vs no } \\
\text { calcification) }\end{array}$ & 0.4528 & 0.1800 & 0.1122 \\
\hline Stool artifact (vs no stool artifact) & 0.7174 & 0.2310 & $0.002 I^{*}$ \\
\hline
\end{tabular}

Note: *Indicates significant difference.

Abbreviations: TRUS, transrectal ultrasonography of the prostate; $\beta$, regression coefficient; SE, standard error; BMI, body mass index; PSA, prostate-specific antigen; vs, versus.

patients to observe real-time images during the procedure reduced patient anxiety; thus, they experienced less pain. The study of Tarhan et al also revealed that video-based education about the procedure of TRUS biopsy can diminish patient anxiety. ${ }^{20}$ Therefore, we suggest that a detailed explanation and the ability to observe real-time images of the procedure can significantly reduce the pain of an anxious patient.

There were some flaws and limitations in our study that are worth mentioning. First, some of the patients who received the examination potentially had prostate cancer. We did not further discuss whether prostate cancer would affect the degree of pain experienced by the patients. Second, although our study confirmed that anal surgical history was one of the independent factors influencing the pain associated with TRUS, some of the given history mainly relied on self-reporting rather than an accurate medical chart review. We were unable to understand how the pain associated with TRUS was affected by different anal surgical procedures, for example, hemorrhoidectomy, hemorrhoid ligation, fistulectomy, or fistulotomy. Last but not least, the diagnosis of external hemorrhoids was made by the inspection of an urologist rather than an anorectal specialist. As a result, the high incidence of external hemorrhoids among our patients was questionable.

\section{Conclusion}

Although a TRUS examination is uncomfortable for patients, we revealed that most of the study patients tolerated the pain and required no anesthesia during the procedure. However, $21.49 \%$ and $12.05 \%$ of the patients reported moderate pain and severe pain, respectively. Our study identified various independent factors influencing the pain associated with TRUS: prostate sagittal length, previous TRUS experience, external hemorrhoids, anal surgical history, and stool accumulation in the rectum. The aforementioned methods enable reducing discomfort. Furthermore, alleviating patient anxiety by providing a detailed explanation before the procedure and allowing patients to observe real-time images can reduce the pain.

\section{Acknowledgments}

This research was supported by grants from the Chang Gung Memorial Hospital (CMRPG3D0311, CMRPG392163, BMRP260) and the National Science Council, Taiwan (NSC 101-2314-B-182A-099-MY3).

\section{Disclosure}

The authors report no conflicts of interest in this work.

\section{References}

1. Watanabe H, Igari D, Tanahasi Y, et al. Development and application of new equipment for transrectal ultrasonography. JClin Ultrasound. 1974;2: 91-98.

2. Alkan I, Türkeri L, Biren T, et al. Volume determinations by transrectal ultrasonography in patients with benign prostatic hyperplasia: correlation with removed prostate weight. Int Urol Nephrol. 1996;28:517-523.

3. Terris MK, Stamey TA. Determination of prostate volume by transrectal ultrasound. J Urol. 1991;145:984-987.

4. Van Praet C, Decaestecker K, Hoebeke P, et al. Correlation between prostate volume measured by transrectal and suprapubic ultrasound: can we do without TRUS? Global congress on Prostate Cancer, Marseille, France, 13-14 June 2013.

5. Seymour H, Perry MJA, Lee-Elliot C, et al. Pain after transrectal ultrasonography-guided prostate biopsy: the advantages of periprostatic local anaesthesia. BJU Int. 2001;88:540-544. 
6. von Knobloch R, Weber J, Varga Z, Feiber H, Heidenreich A, Hofmann R. Bilateral fine-needle administered local anaesthetic nerve block for pain control during TRUS-guided multi-core prostate biopsy: a prospective randomised trial. Eur Urol. 2002;41:508-514.

7. Irani J, Fournier F, Bon D, Gremmo E, Dore B, Aubert J. Patient tolerance of transrectal ultrasound-guided biopsy of the prostate. Br J Urol. 1997;79:608-610.

8. Huskisson EC. Measurement of pain. Lancet. 1974;2:1127-1131.

9. Hawker GA, Mian S, Kendzerska T, French M. Measures of adult pain: visual analog scale for pain (vas pain), numeric rating scale for pain (nrs pain), mcgill pain questionnaire (mpq), short-form mcgill pain questionnaire (sf-mpq), chronic pain grade scale (cpgs), short form-36 bodily pain scale (sf-36 bps), and measure of intermittent and constant osteoarthritis pain (icoap). Arthritis Care Res. 2011;63(S11): S240-S252.

10. Philip J, McCabe JE, Roy SD, Samsudin A, Campbell IM, Javle P. Site of local anaesthesia in transrectal ultrasonography-guided 12-core prostate biopsy: does it make a difference? BJU Int. 2006;97(2): 263-265.

11. Rodriguez A, Kyriakou G, Leray E, Lobel B, Guille F. Prospective study comparing two methods of anaesthesia for prostate biopsies: apex periprostatic nerve block versus intrarectal lidocaine gel: review of the literature. Eur Urol. 2003;44(2):195-200.
12. Bennett RC, Duthie HL. The functional importance of the internal sphincter. Br J Surg. 1964;51:355-357.

13. Lestar B, Penninckx F, Kerremans R. The composition of anal basal pressure. An in vivo and in vitro study in man. Int J Colorectal Dis. 1989; 4(2):118-122.

14. Frenckner B, Euler CV. Influence of pudendal block on the function of the anal sphincters. Gut. 1975;16(6):482-489.

15. Gibbons CP, Trowbridge EA, Bannister JJ, Read NW. Role of anal cushions in maintaining continence. Lancet. 1986;1(8486):886-888.

16. Jacobs D. Clinical practice. Hemorrhoids. N Engl J Med. 2014;371(10): 944-951.

17. Sayfan J, Becker A, Koltun L. Sutureless closed hemorrhoidectomy: a new technique. Ann Surg. 2001;234(1):21-24.

18. Theerapol A, So BY, Ngoi SS. Routine use of setons for the treatment of anal fistulae. Singapore Med J. 2002;43(6):305-307.

19. Czarnecki ML, Turner HN, Collins PM, Doellman D, Wrona S, Reynolds J. Procedural pain management: a position statement with clinical practice recommendations. Pain Manag Nurs. 2011;12(2):95-111.

20. Tarhan H, Cakmak O, Unal E, et al. The effect of video-based education on patient anxiety in men undergoing transrectal prostate biopsy. Can Urol Assoc J. 2014;8:11-12.
Patient Preference and Adherence

\section{Publish your work in this journal}

Patient Preference and Adherence is an international, peer-reviewed, open access journal that focuses on the growing importance of patient preference and adherence throughout the therapeutic continuum. Patient satisfaction, acceptability, quality of life, compliance, persistence and their role in developing new therapeutic modalities and compounds to optimize

\section{Dovepress}

clinical outcomes for existing disease states are major areas of interest for the journal. This journal has been accepted for indexing on PubMed Central. The manuscript management system is completely online and includes a very quick and fair peer-review system, which is all easy to use. Visit http://www. dovepress.com/testimonials.php to read real quotes from published authors. 